\title{
Efectos del huracán "Pauline" (1997) sobre la fauna asociada a la planta Eichhornia crassipes en la Laguna Coyuca, Pacífico Sur de México
}

\section{Ramiro Román-Contreras ${ }^{1}$, Arturo Rocha-Ramírez ${ }^{2} \&$ Sergio Cházaro-Olvera²}

1. Laboratorio de Carcinoparasitología, Instituto de Ciencias del Mar y Limnología, Universidad Nacional Autónoma de México (UNAM), Apartado Postal 70-305, México D.F. 04510, México; ramiror@mar.icmyl.unam.mx

2. Laboratorio de Ecología, Facultad de Estudios Superiores Iztacala, Universidad Nacional Autónoma de México (UNAM), Av. de los Barrios 1, Los Reyes Iztacala, Apartado Postal 314, Tlalnepantla, Edo. de México, C.P. 54090, México; arocha@servidor.unam.mx; chazaro@campusiztacala.unam.mx

\section{Recibido 22-III-2007. Corregido 05-X-2007. Aceptado 19-XI-2007.}

\begin{abstract}
Effects of hurricane "Pauline" (1997) on the fauna associated with the plant Eichhornia crassipes in Laguna Coyuca, South Pacific of Mexico. Reports on the effects of hurricanes on marine and coastal environments often deal with coral reefs, but little is known about their effect on the communities associated with the water hyacinth, Eichhornia crassipes. From January 1997 (pre-hurricane) through April 1998 (post-hurricane) we made montly collections of fauna in E. crassipes roots from Laguna Coyuca, Mexico $\left(17^{\circ} 00^{\prime}-16^{\circ} 54^{\prime} \mathrm{N}\right.$, $\left.99^{\circ} 58^{\prime}-100^{\circ} 05^{\prime} \mathrm{W}\right)$. The hurricane affected Coyuca on October $9^{\text {th }}$, 1997 and caused mortalities of that fauna. During the three subsequent months the absence of E. crassipes and its associated fauna in the study area was evident, but in January 1998, we found a partial reestablishment of E. crassipes and its associated fauna. Four months later, this community was almost back to pre-hurricane levels. Rev. Biol. Trop. 56 (2): 603-611. Epub 2008 June 30.
\end{abstract}

Key words: Hurricane "Pauline", effects, associated fauna, Eichhornia crassipes, coastal lagoon.

Los efectos de huracanes en distintas áreas geográficas y comunidades de ambientes marinos y costeros han sido reportados desde hace muchos años. Entre ellos las evaluaciones en los arrecifes de coral son las que mayor atención han recibido (Stoddart 1962, Flood y Jell 1977, Kaufman 1983, Williams 1984, Laboute 1985, Laboute 1986, Harmelin-Vivien y Laboute 1986, Yoshioka y Yoshioka 1987, Fenner 1991, Moran y Reaka-Kudla 1991); las comunidades de peces y otra fauna marina (Robins 1957, Lassig 1983, Pfeffer y Tribbles 1985, Letourneur et al. 1993); así como los efectos de tormentas sobre ambientes sedimentarios y cambios físicos en algunas localidades (Ball et al.1963, Vermeer 1963) también han sido tratados. En el presente trabajo se presentan resultados de observaciones que sobre la fauna asociada al lirio acuático Eichhornia crassipes (Martens) Solms-Laubenf tuvo el huracán "Pauline" a su paso sobre la laguna costera de Coyuca, ubicada aproximadamente a $10 \mathrm{~km}$ del puerto de Acapulco, sur del Pacífico de México.

Estadísticas de la tormenta: con base en los registros del Tropical Prediction Center, National Weather Service (NOAA) de Miami (Lawrence 1997a, b, Anónimo 1997) el 5 de octubre de 1997 se formó la depresión tropical No. 18-E de la temporada en el Pacífico nor-oriental que se localizó a $425 \mathrm{~km}$ al sur del Golfo de Tehuantepec, sur del Pacífico de México (Fig. 1). En la madrugada del día 6 la depresión tropical se desarrolló como tormenta tropical a $395 \mathrm{~km}$ al suroeste de Tapachula, Chiapas, a la que se nombró "Pauline". El día 7 el huracán alcanzó vientos máximos sostenidos de $215 \mathrm{~km} / \mathrm{h}$ y rachas de $240 \mathrm{~km} / \mathrm{h}$, por lo que 


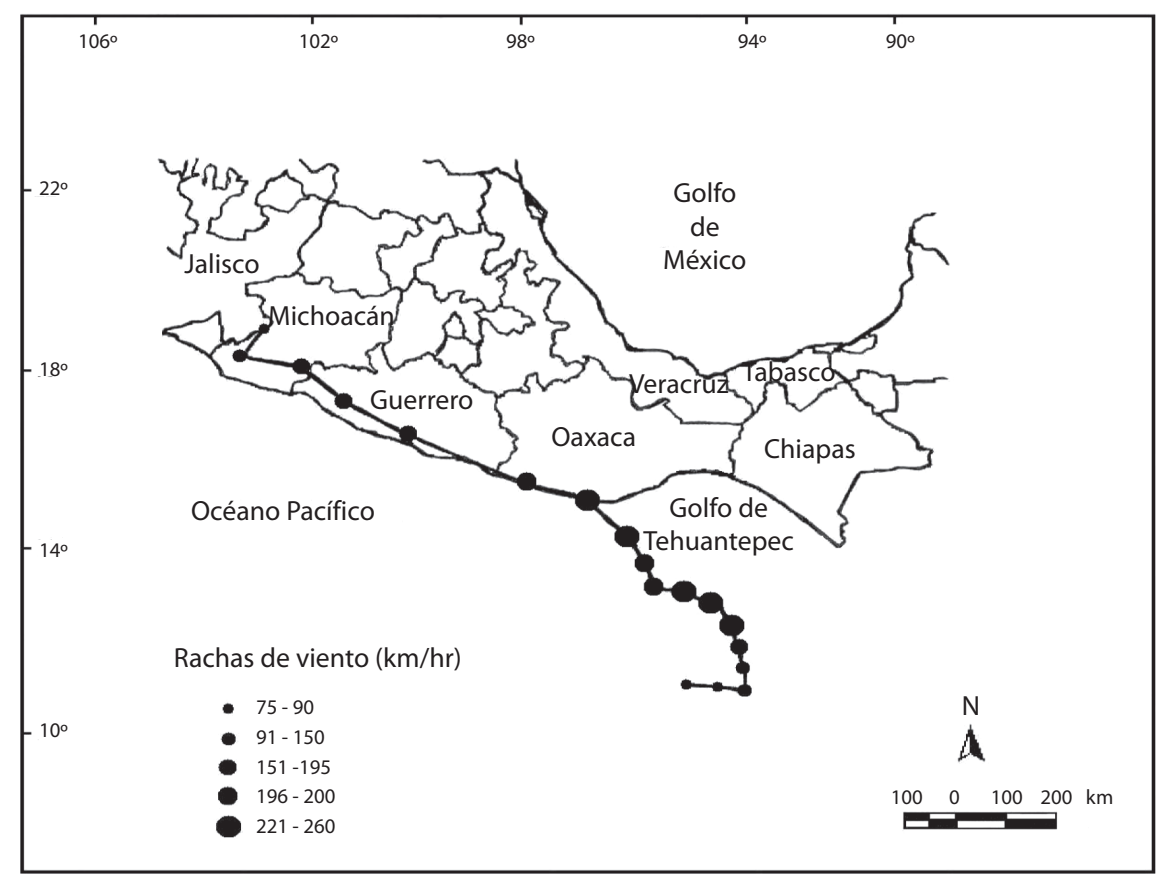

Fig. 1. Trayectoria del huracán "Pauline" en el Pacífico sur de México entre el 5 y 10 de octubre de 1997. (Modificado de Anónimo 1997, y Anónimo 2002).

presentó la categoría 4 en la escala de intensidad Saffir-Simpson. Por la tarde "Pauline" se debilitó a categoría 3 con vientos hasta de $185 \mathrm{~km} / \mathrm{h}$. El día 8, antes de tocar la costa, "Pauline" recuperó la categoría 4 alcanzando vientos máximos de $210 \mathrm{~km} / \mathrm{h}$ y rachas de 260 $\mathrm{km} / \mathrm{h}$ a $100 \mathrm{~km}$ al sur-suroeste de Huatulco, Oaxaca, en la costa mexicana. Por la tarde el huracán entró a tierra firme con vientos máximos de $185 \mathrm{~km} / \mathrm{h}$ y rachas hasta de 240 $\mathrm{km} / \mathrm{h}$ (Anónimo 1997). Desde el momento en que tocó tierra, el huracán mantuvo su desplazamiento sobre la costa sur de México y su trayectoria fue predominantemente hacia el noroeste internándose en la costa del estado de Guerrero (Fig. 1). En la madrugada del día 9, el "ojo" del huracán se localizó a solo $30 \mathrm{~km}$ al nor-noroeste de Acapulco registrando vientos máximos sostenidos de $165 \mathrm{~km} / \mathrm{h}$ y rachas que alcanzaron hasta $200 \mathrm{~km} / \mathrm{h}$.

Por efectos de la orografía de la región los bordes del ojo del huracán golpearon fuertemente con lluvias intensas al puerto de Acapulco. Durante el día 9 "Pauline" siguió avanzando sobre tierra con dirección oeste-noroeste pasando a $40 \mathrm{~km}$ al norte de Zihuatanejo, Guerrero, con vientos máximos de $150 \mathrm{~km} / \mathrm{h}$ y rachas de $195 \mathrm{~km} / \mathrm{h}$ y por la tarde, a $45 \mathrm{~km}$ al nor-noroeste del puerto de Lázaro Cárdenas, Michoacán, con vientos de 140 km/h y rachas de $165 \mathrm{~km} / \mathrm{h}$. Al avanzar sobre la zona montañosa de la región "Pauline" empezó a debilitarse convirtiéndose al anochecer en tormenta tropical. Ésta siguió su desplazamiento sobre tierra debilitándose paulatinamente, por lo que en la madrugada del día 10 se degradó a depresión tropical aproximadamente a $30 \mathrm{~km}$ al suroeste de la ciudad de Uruapan, Michoacán, disipándose finalmente al sur-suroeste de Guadalajara, Jalisco, en el centro-occidente de México.

Hidrología de la laguna: la cuenca lagunar de Coyuca es una de las 123 lagunas costeras de México (Lankford 1977) ubicada en la 
porción centro-sur del Pacífico mexicano, y se caracteriza por ser de boca efímera y estar separada del mar por una barrera de arena (YáñezArancibia 1976, Guzmán-Arroyo et al. 1986). Tiene alrededor de $10.5 \mathrm{~km}$ de longitud y 4.5 $\mathrm{km}$ en su parte más ancha y una profundidad máxima de $18 \mathrm{~m}$ en la región central (Fig. 2) (Ramírez-Granados 1952). Aproximadamente el $50 \%$ del área lagunar presenta profundidades menores a $4 \mathrm{~m}$; en su porción occidental la cuenca recibe los aportes del río Coyuca, que tiene una descarga anual de $975.6 \times 10^{6} \mathrm{~m}^{3}$ de agua al año que proviene de las montañas (Guzmán-Arroyo et al. 1986) y es la fuente de mayor aporte a la laguna. Esta recibe también las aguas del Río Conchero ubicado en la parte sur-oriental, cuya reducida aportación se limita a la época de lluvias (Román-Contreras 1991, Fonseca y Lozada-Pérez 1993). Otros arroyos temporales y escurrimientos de menor volumen descargan sus aguas a la cuenca lagunar (Ramírez-Granados 1952, Román-Contreras 1991, Contreras 1993), lo que contribuye a que la laguna se llene durante la época de mayor precipitación.

Desde hace varias décadas la barrera de arena que separa a laguna del mar se abre frente a la desembocadura del Río Coyuca durante dos períodos al año por un canal de aproximadamente $10 \mathrm{~m}$, aunque generalmente su apertura es inducida; este fenómeno tiene lugar cuando la laguna alcanza los máximos niveles, lo que sucede entre agosto-septiembre y entre noviembre-diciembre, pero la influencia marina en el canal adyacente a la boca de la laguna es escasa lo que determina condiciones oligohalinas en el área interna de la desembocadura.

\section{MATERIALES Y MÉTODOS}

Sitio de recolecta: desde enero de 1997 (pre-huracán) y hasta abril de 1998 (posthuracán) se realizaron recolectas mensuales de la fauna asociada al sistema radicular del lirio acuático E. crassipes en un sitio que se caracteriza por la presencia permanente de lirio acuático, ubicado en la porción media interna de la barra de la laguna Coyuca $\left(17^{\circ} 00^{\prime}-16^{\circ} 54^{\prime}\right.$ N, 99 $58^{\prime}-100^{\circ} 05^{\prime}$ W) (Fig. 2). La temperatura y la salinidad fueron registradas in situ con un termómetro portátil y un salinómetro manual AO, respectivamente. Para la recolecta de los organismos del sistema radicular se introdujo bajo cada planta una red de plancton con abertura de malla de 250 micras; las plantas fueron retiradas completas del agua, colocadas en un

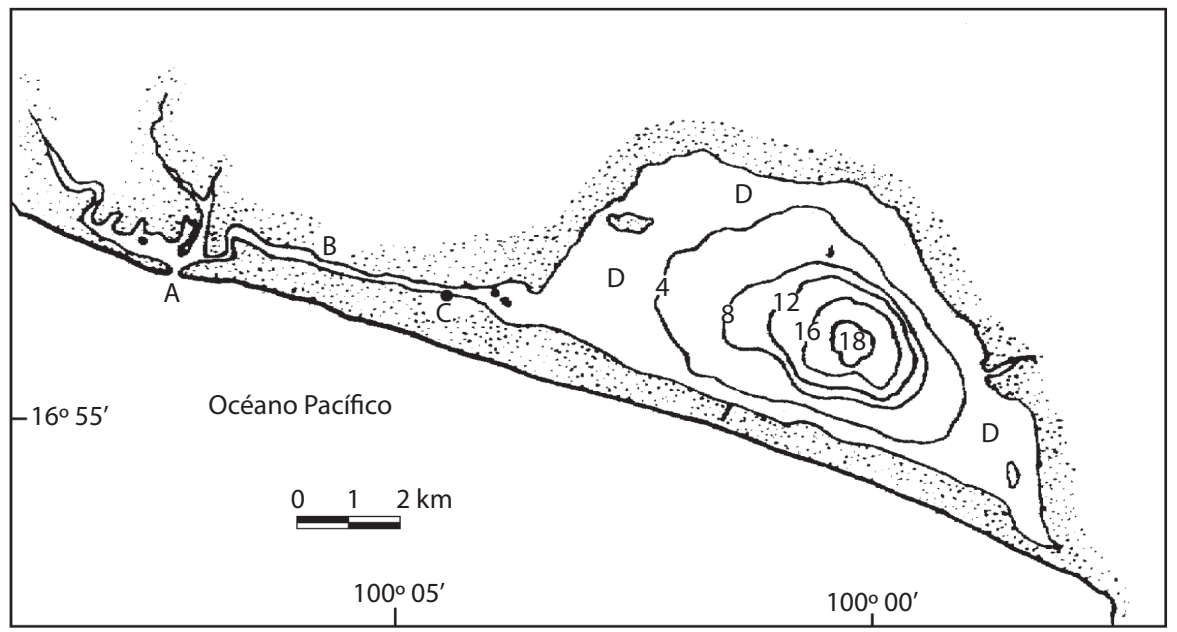

Fig. 2. Topografía y batimetría de la Laguna Coyuca, Guerrero, Pacífico sur de México; A) Boca de la barra; B) Canal de la laguna; C) Estación de colectas; D) Cuenca lagunar. (Modificado de Ramírez-Granados 1952). 
recipiente con agua y sacudidas vigorosamente para desprender los organismos de las raíces hasta cerciorarse visualmente de su ausencia. Los organismos fueron concentrados en bolsas de plástico, preservados en formalina al $4 \% \mathrm{y}$ transportados al laboratorio donde fueron lavados con agua corriente é identificados hasta el nivel taxonómico mas bajo posible.

\section{RESULTADOS}

Registros faunísticos pre-huracán: durante los meses anteriores al huracán se recolectaron crustáceos decápodos como juveniles de Macrobrachium tenellum (Smith), Potimirim glabra (Kingsley), Glyptograpsus impressus Smith y Litopenaeus vannamei (Boone). Entre los peracáridos, anfípodos de las familias Corophiidae, Talitridae, Gammaridae y Terebridae; además de Anopsilana oaxaca Carvacho y Haasmann y Cassidinidea mexicana Hendrickx y Espinosa-Pérez, que fueron particularmente abundantes entre las raíces del lirio acuático; además se recolectó un espécimen de M. tenellum infestado por Probopyrus pacificensis Román-Contreras, y un individuo del género Tanais Latreille. Entre la entomofauna asociada a $E$. crassipes estuvieron presentes adultos de Tropisternus mexicanus Laporte y Belostoma aztecus L. y representantes de los géneros Aphylla Selys, Coryphaeschna Williamson, Telebasis Selys, Abedus Stal, Ranatra Fabricius y Rheumatobates Bergroth, así como larvas y adultos de Berosus Leach y Megadytes Sharp. Neritina latissima Broderip, Biomphalaria Preston y Physella Haldeman, y algunos individuos de la familia Hydrobiidae también fueron registrados como representantes de los moluscos; y juveniles de Astyanax fasciatus (Cuvier), Bathygobius soporator (Cuvier y Valiencennes) y Gobionellus microdon (Gilbert), entre los peces.

Durante 1997 la boca de la laguna fue abierta en junio y la corriente lagunar permaneció drenando hacia el océano durante las dos siguientes semanas, como ha sucedido desde hace varias décadas como consecuencia del aporte del río Coyuca durante los meses anteriores al período lluvioso. Antes de la apertura de la barra arenosa el lirio acuático ocupó amplias zonas de inundación del canal aledaño en el área interna de la barra, así como otras zonas de la laguna.

La apertura de la barra en el segundo periodo fue inducida por el huracán "Pauline" y sucedió dos semanas después de su paso por el área, como resultado del volumen extraordinario de pluviosidad registrado en la Estación Meteorológica de Coyuca de Benítez ubicada aproximadamente $8 \mathrm{~km}$ río arriba de la barra, lo que provocó que el vaso lagunar se llenara nuevamente hasta su nivel máximo acelerando el proceso de la segunda apertura. La boca de la laguna frente al río Coyuca (Fig. 2) fue abierta en esa ocasión en forma natural y tuvo una dimensión aproximada de $50 \mathrm{~m}$; el rápido vaciado provocó el acarreo del lirio acuático mar afuera y su acumulación en los bordes internos adyacentes a la desembocadura donde se depositaron grandes volúmenes; la boca se cerró totalmente hasta finales de diciembre, lo que significó un periodo extraordinario de casi tres meses de comunicación con el mar.

Los vientos que prevalecieron durante el huracán ocasionaron la defoliación de las áreas de manglar presentes en las riberas lagunares (Rhizophora mangle L., Laguncularia racemosa (L.) Gaertner, Conocarpus erecta L., (Ramírez-Granados 1952, Román-Contreras 1991, Fonseca y Lozada-Pérez 1993), siendo la defoliación de estas especies arbóreas más notoria en las zonas de mayor exposición al fenómeno.

Registros faunísticos post-huracán: en enero de 1998 se observó el inicio de la recuperación de parches de E. crassipes en la zona de estudio y se obtuvieron los primeros registros de la fauna asociada. Las especies recolectadas fueron A. fasciatus, B. (ca.) soporator, B. aztecus, M. tenellum, P. glabra, A. oaxaca, N. latissima, Biomphalaria sp., Ranatra sp. y anfípodos de las familias Talitridae y Gammaridae.

Para abril de 1998 se observó una marcada recuperación de la población de lirio acuático en la laguna y al mismo tiempo de la fauna 
asociada; además de las especies registradas en enero anterior se recolectaron individuos de $G$. microdon, G. impressus, C. mexicana, Berosus sp., Megadytes sp., Aphylla sp., Abedus sp. y Physella sp.; y nuevamente representantes de las familias Talitridae y Gammaridae, además de Corophiidae y Terebridae.

\section{DISCUSIÓN}

El huracán "Pauline" fue el más intenso que azotó las costas del estado de Guerrero después del "Madeline" (29 de Septiembre al 8 de Octubre de 1976), que presentó vientos máximos de 232 $\mathrm{km} / \mathrm{h}$ en la costa del estado. "Pauline" provocó una precipitación extraordinaria en el área de $411.2 \mathrm{~mm}$ en tan solo $24 \mathrm{~h}$, que comparada con la máxima histórica de $384 \mathrm{~mm}$ del 16 de junio de 1974 en Acapulco, Guerrero, representó un nuevo récord histórico (Anónimo 1997, Lawrence 1997b). El valor de pluviosidad durante el huracán "Pauline" también fue relativamente cercano al promedio de precipitación anual de 487.8 mm registrado en la estación meteorológica de Coyuca de Benítez durante la década de 1970-80 (Guzmán-Arroyo et al. 1986).

Diversos autores han señalado que tanto la diversidad bentónica como la producción de la laguna de Coyuca es baja (Stuardo y Martínez 1975, Stuardo y Villarroel 1976, Yáñez-Arancibia 1978a, Román-Contreras 1991). Sin embargo, estudios mas recientes (Rocha-Ramírez et al. 2002) y resultados del presente trabajo, han revelado la presencia de gran variedad de organismos asociados a las raíces de E. crassipes, que son componente importante de los procesos ecológicos del sistema y pesquerías locales.

Los invertebrados en el área de estudio están representados por varios grupos pero algunas especies de crustáceos como $M$. tenellum se encuentran en gran abundancia y son más conspicuas que otras (Román-Contreras 1976, 1991); esta especie sostiene una pesquería artesanal y regional de mediana importancia mientras que de Macrobrachium americanum Bate, otra especie de langostino presente en el área, se recolectaron pocos individuos juveniles.
Ramírez-Granados (1952), 45 años antes del huracán "Pauline" registró la presencia de cladóceros, ostrácodos, copépodos, isópodos y anfípodos; y entre los decápodos, peneidos, palemónidos y braquiuros como Goniopsis pulchra (Lockington) y Callinectes toxotes Ordway. Por su parte, Stuardo y Martínez (1975) agregaron L. vannamei a la lista decápodos de la laguna. Román-Contreras (1991) recolectó y confirmó la presencia de jóvenes de $L$. vannamei y agregó a la lista a Farfantepenaeus brevirostris Kingsley y Farfantepenaeus californiensis Holmes, especies que indudablemente ingresan a la laguna en sus fases larvales durante el breve periodo anual de apertura de la barra.

Otros registros de decápodos adultos fueron Callinectes arcuatus Ordway, Sesarma sulcatum Smith y G. impressus, así como dos especies de atyidos: Atya margaritacea A. Milne-Edwards, y juveniles de P. glabra que se recolectaron en muestras post-huracán entre las raíces del lirio acuático cerca de la desembocadura del río Coyuca como resultado del arrastre a esa área, dado que en la parte alta de la cuenca existen poblaciones importantes de ambas especies (Martínez-Mayén et al. 2000, Martínez-Mayén y Román-Contreras 2000, Martínez-Mayén y Román-Contreras 2003, Martínez-Mayén et al. 2004).

Ramírez-Granados (1952) mencionó la recolección de isópodos en muestras tomadas en el fondo y en las orillas de la laguna sin mencionar las especies a que pertenecían estos organismos. Román-Contreras (1976, 1993a, 1993b), por su parte, recolectó numerosos individuos de $P$. pacificensis, bopyrido branquial que infesta a $M$. tenellum y se distribuye desde El Salvador, Centro América (Holthuis 1954), hasta la región norte del Pacífico mexicano (Román-Contreras 2004).

Stuardo y Martínez (1975) y RománContreras (1991) reportaron la presencia del isópodo Cirolana sp. en la laguna sin determinar la especie de los organismos recolectados; Rocha-Ramírez et al. (2002) por su parte, describieron y calcularon la fecundidad de $A$. oaxaca recolectados de las raíces de 
E. crassipes en los períodos pre- y post-huracán; los autores concluyeron que $A$. oaxaca está presente durante todo el año y que su presencia en el área es una de las mas notables entre los isópodos.

Montoya (2003) comprobó que las raíces de E. crassipes albergan a una comunidad de invertebrados acuáticos numéricamente importante que a su vez sirven de alimento a otras especies; entre otros, las especies del género Macrobrachium son de los pocos invertebrados que se alimentan directamente de las raíces de E. crassipes (Green et al.1976, Montoya 2003) amén de la protección que les proporciona. Román-Contreras (1991), Montoya (2003) y Rocha-Ramírez et al. (2002) han documentado que juveniles del género Macrobrachium son frecuentes entre las raíces de E. crassipes $\mathrm{y}$ de la vegetación acuática sumergida. La vegetación acuática es, además, un ambiente propicio para el establecimiento de poblaciones de insectos acuáticos tanto en estados larvales como de adultos.

Ramírez-Granados (1952) citó a los hemípteros acuáticos como poco abundantes y a los dípteros de la familia Chironomidae como "abundantísimos entre la vegetación ribereña y en el contenido del tubo digestivo de ciertos peces". En tanto, Román-Contreras (1991) y Rocha-Ramírez et al. (2002) recolectaron entre las raíces y follaje de E. crassipes varias especies de entomofauna entre las que registraron, después del paso del huracán, a T. mexicanus adultos, larvas y adultos de Berosus sp., larvas de Megadytes sp., adultos de Aphylla sp., Coryphaeschna sp., Telebasis sp., B. aztecus y Belostoma sp., Abedus sp., Ranatra sp. y posiblemente Rheumatobates sp.; además de algunas especies de dípteros que no fueron determinadas.

Stuardo y Villarroel (1976) estudiaron aspectos biológicos y distribución de los moluscos de las lagunas costeras del estado de Guerrero, pero inexplicablemente la laguna Coyuca no fue incluida en sus estudios, por lo que la información sobre este grupo es muy reducida. Especies vivas de moluscos fueron recolectadas por Román-Contreras (1991) y por Rocha Ramírez et al. (2002) entre las raíces de E. crassipes y otro tipo de vegetación acuática de la laguna. Estas incluyeron representantes de las familias Hydrobiidae, Physidae (Physella sp.) y representantes de las familias Planorbidae (Biomphalaria sp.) con reducida presencia; así como especies de la familia Neritidae ( $N$. latissima), cuya abundancia fue relativamente elevada.

Ramírez-Granados (1952) señaló la presencia de 18 especies de peces adultos en la localidad pero estudios posteriores (YáñezArancibia 1978a, b) reportaron información adicional sobre otras especies presentes en la laguna Coyuca. Yáñez-Arancibia (1978a) realizó un estudio sobre los patrones y la estructura trófica de comunidades nectónicas en lagunas costeras del Pacífico de México sin especificar aquéllas en las que trabajó. En una investigación posterior, Yáñez-Arancibia (1978b) puntualizó las localidades y determinó 25 especies de peces recolectados en la laguna Coyuca durante septiembre y octubre de 1973 y 1974; para las recolectas, dicho autor utilizó diferentes implementos pesqueros por lo que las muestras pudieron haber sido selectivas recolectando solamente individuos adultos.

En estudios anteriores al huracán "Pauline" Román-Contreras (1991) registró la presencia de 13 especies de peces juveniles como fauna de acompañamiento del camarón de agua dulce M. tenellum en la misma laguna recolectados mediante redes de cuchara, método que pudo haber influido en la obtención de sólo el $50 \%$ de las especies reportadas por Yáñez-Arancibia (1978b) al escapar los peces de mayor tamaño. Es notorio que a pesar del número de especies reportadas antes del huracán "Pauline" por Ramírez-Granados (1952), Yáñez-Arancibia (1978b) y Román-Contreras (1991), en las recolectas post-huracán solamente se hayan registrado13 especies de peces juveniles, lo que sugiere que el fenómeno atmosférico influyó negativamente en la presencia de los jóvenes de peces asociados a las raíces de E. crassipes.

Aunque en general se reconoce que los huracanes son eventos catastróficos que alteran severamente los ecosistemas naturales (Mallin 
et al. 1999), existen evidencias de que la acción de estos fenómenos no siempre son adversos a las comunidades de peces, ya que ni Houde et al. (2005) ni Locascio y Mann (2005) observaron efectos negativos inmediatos en las poblaciones de peces por los huracanes "Isabel" y "Charley" a su paso por la Bahía Chesapeake y Charlotte Harbor, respectivamente. Por el contrario, Houde et al. (2005) documentaron mayor abundancia de jóvenes de algunas especies de peces en los muestreos post-huracán "Isabel" durante 2003, mientras que Locascio y Mann (2005) supusieron que una baja en las concentraciones de oxígeno disuelto por efecto retardado de "Charley" pudo haber afectado la actividad reproductora de los peces.

Covich et al. (1991), después del paso del huracán "Hugo" por el bosque experimental de Luquillo, Puerto Rico, observaron una abundancia inusitada de Atya lanipes Holthuis que no habían observado anteriormente en esta especie de camarones, lo que atribuyeron tanto a su migración río arriba como al aumento de la disponibilidad de recursos alimenticios acarreados por el fenómeno.

La disminución en el número de especies de peces juveniles en las recolectas post huracán en la laguna Coyuca puede atribuirse a la disminución de E. crassipes en el área de estudio, cuya presencia juega un papel importante como sitio de reproducción, alimentación y protección contra depredadores.

Si bien "Pauline" tuvo efectos negativos temporales sobre la abundancia de la fauna asociada a E. crassipes, y tomando en consideración el hábitat dulceacuícola del lirio y su alta capacidad de regeneración, era de esperarse la sucesiva repoblación de la fauna asociada a esta especie de fanerógama, lo que fue observado pocos meses después pudiendo constatarse la paulatina recuperación de la mayoría de las especies asociadas al lirio acuático registradas con anterioridad al huracán, por lo que se concluye que el tiempo de recuperación de la fauna asociada a las raíces de E. crassipes en la laguna Coyuca puede considerarse de corto plazo en comparación con otras comunidades y ambientes costeros en los que tanto el impacto de los huracanes como el tiempo de recuperación son, en muchos de los casos, de mayor magnitud.

Finalmente, la presencia de especies adicionales de diferentes grupos en el sistema radicular de E. crassipes en la laguna Coyuca evidenció la enorme riqueza de diversos grupos faunísticos que alberga $E$. crassipes en sus raíces, y la necesidad de realizar estudios mas amplios con diferentes métodos de recolecta, con la finalidad de conocer con mayor amplitud los componentes biológicos presentes en los ecosistemas lagunares antes y después de fenómenos naturales como el huracán "Pauline".

\section{AGRADECIMIENTOS}

Los autores agradecen a M. MartínezMayén, del Instituto de Ciencias del Mar y Limnología de la UNAM, por su apoyo técnico durante el trabajo de campo y la preparación de este trabajo. Igualmente, a tres revisores anónimos cuyas sugerencias ayudaron a mejorar el manuscrito.

\section{RESUMEN}

Observaciones sobre los efectos que el huracán "Pauline" produjo a su paso por la laguna Coyuca, sur del Pacífico de México la madrugada del 9 de octubre de 1997, indican que el disturbio tropical ocasionó en el área la desaparición temporal de la fauna asociada a las raíces del lirio acuático en relación a la fauna registrada durante los meses anteriores al fenómeno; durante los tres meses posteriores se registró la disminución de E. crassipes en el área de estudio. En enero de 1998 se observó el inicio de su recuperación y se recolectaron los primeros organismos asociados a sus raíces; cuatro meses más tarde el lirio acuático y su fauna asociada casi alcanzaban los mismos niveles registrados antes del huracán.

Palabras clave: Huracán "Pauline", efectos, fauna asociada, Eichhornia crassipes, laguna costera.

\section{REFERENCIAS}

Anónimo. 1997. "Huracán Pauline” del Océano Pacífico (5 octubre-10 octubre) 1997. Comisión Nacional del Agua, Servicio Meteorológico Nacional de México, México, D.F. 
Anónimo. 2002. Huracán Paulina, su paso por costas mexicanas. Instituto de Geografía, UNAM y CONABIO, México D.F., México.

Ball, M.M., E.A. Shinn \& W. Stokman. 1963. Geologic record of hurricanes. Abstracts Amer. Asso. Petrol. Geol. Soc. Econ. Paleontol. Mineral \& Mid-Western Soc. Explor. Geophys. Papers, National Convention, Houston, Texas, EEUU.

Contreras, E.F. 1993. Ecosistemas Costeros Mexicanos. CONABIO-Univ. Autónoma Metropolitana, Iztapalapa, México D.F., México.

Covich, A.P., T.A. Crowl, S.L. Johnson, D. Varza \& D.L. Certain. 1991. Post-hurricane Hugo increases in Atyid shrimp abundances in a Puerto Rican stream. Biotropica 23: 448-454.

Fenner, D.P. 1991. Effects of hurricane Gilbert on coral reef-fishes and sponges at Cozumel, Mexico. Bull Mar. Sci. 48: 719-730.

Flood, P.G. \& J.S. Jell. 1977. The effect of cyclone David (January, 1976) on the sediment distribution patterns on Heron reef. Great Barrier Reef, Australia. Proc. $3^{\text {rd }}$ Int. Coral Reef Symp. Miami 2: 120-125.

Fonseca, R.M. \& L Lozada-Pérez. 1993. Estudios Florísticos en Guerrero. In N. Diego-Pérez \& R.M. Fonseca (eds.). No. 1. Laguna de Coyuca. Prensas de Ciencias. Facultad de Ciencias, UNAM, México D.F., México.

Green, J., S.A. Corbet, E. Watts, \& O.B. Lan. 1976. Ecological studies on Indonesian lakes. Overturn and restratification of Ranu Lamongan. J. Zool. 180: 315-354.

Guzmán-Arroyo, M., S. Mañon-Ontiveros \& M.A. OrtizPérez. 1986. Afinidad limnológica del sistema lagunar costero del estado de Guerrero, México. Bol. Inst. Geog. UNAM 16: 61-76.

Harmelin-Vivien, M.L. \& P. Laboute. 1986. Catastrophic impact of hurricanes on atoll outer reef slopes in the Tuamotu (French Polynesia). Coral Reefs 5: 55-62.

Holthuis, L.B. 1954. On a collection of Decapod Crustacea from the Republic of El Salvador (Central America). Zool. Verh. 23: 1-43.

Houde, E.D., J. Bichy \& S. Jung. 2005. Effects of hurricane Isabel on fish populations and communities in Chesapeake Bay, p. 193-199. In K.G. Sellner (ed.). Hurrican Isabel in Perspective. Proc. Conf. CRC Publ. 5, Boca Raton Florida, EEUU.
Kaufman, L.S. 1983. Effects of hurricane Allen on reef fish assemblages near Discovery Bay. Coral Reefs 2: 43-47.

Laboute, P. 1985. Evaluation of damage done by the cyclones of 1982-83 to the outer slopes of the Tuamotu and Takapoto atolls, Tuamotu Archipielago. Proc. $5^{\text {th }}$ Int. Coral Reef Symp., Thaiti 3: 323-329.

Lankford, R.R. 1977. Coastal Lagoons of Mexico. Their origin and classification, p. 182-215. In M. Wiley (ed.). Estuarine Processes. Academic, Nueva York, EEUU.

Lassig, B.R. 1983. The effects of a cyclonic storm on coral reef fish assemblage. Environ. Biol. Fish. 9: 55-63.

Lawrence, M.B. 1997a. Preliminary report Hurricane Pauline 5-10 October 1997. National Hurricane Center. Tropical Prediction Center. Miami, EEUU.

Lawrence, M.B. 1997b. Eastern North Pacific Hurricane Season of 1997. Month. Weather Rev. 127: 2440-2454.

Letourneur, Y., M.L. Harmelin-Vivien \& R Galzin. 1993. Impact of hurricane Firinga on fish community structure on fringing reefs of Reunion Island, S.W. Indian Ocean. Environ. Biol. Fish. 37: 109-120.

Locascio, J.V. \& D.A. Mann. 2005. Effects of hurricane Charley on fish chorusing. Biol. Lett. 1: 362-365.

Mallin, M.A., M.H. Posey, G.C.H. Shank, M.R. McIver, S.H. Ensign \& T.D. Alphin. 1999. Hurricane effects on water quality and benthos in the Cape Fear watershed: natural and anthropogenic impacts. Ecol. Appl. 9. (abstract).

Martínez-Mayén, M. \& R. Román-Contreras. 2000. Aspects of the reproduction of Atya margaritacea A. MilneEdwards, 1864 (Decapoda, Atyidae) in a population from the Mexican Pacific. Crustaceana 73: 913-923.

Martínez-Mayén, M. \& R. Román-Contreras. 2003. Reproducción de Potimirim glabra (Kingsley, 1878) (Crustacea: Decapoda: Atyidae) en el río Coyuca, Guerrero, México, p. 103-115. In M.E. Hendrickx (ed.). Contribuciones al Estudio de los Crustáceos del Pacífico Este 2 (Contributions to the Study of the East Pacific Crustaceans 2). Instituto de Ciencias del Mar y Limnología, UNAM, México D.F., México.

Martínez-Mayén, M., R. Román-Contreras, A. RochaRamírez \& S. Cházaro-Olvera. 2000. Relative growth of Atya margaritacea A. Milne-Edwards, 1864 (Decapoda, Atyidae) from the southern Pacific coast of Mexico. Crustaceana 73: 525-534. 
Martínez-Mayén, M., R. Román-Contreras \& M.A. Martínez-Muñoz. 2004. Growth of fresh water shrimp Potimirim glabra (Kingsley, 1878) (Crustacea: Decapoda: Atyidae) in the Coyuca River, Guerrero, Mexico, p. 189-197. In M.E. Hendrickx (ed.). Contribuciones al Estudio de los Crustáceos del Pacífico Este 3 (Contributions to the Study of the East Pacific Crustaceans 3). Instituto de Ciencias del Mar y Limnología, UNAM. México D.F., México.

Montoya, J.V. 2003. Freshwater shrimps of the genus Macrobrachium associated with roots of Eichhornia crassipes (Water hyacincth) in the Orinoco Delta (Venezuela). Carib. J. Sci. 39: 155-159.

Moran, D.P. \& M.L. Reaka-Kudla. 1991. Effects of disturbance: disruption and enhancement of coral reef crypto-faunal populations by hurricanes. Coral Reefs 9: $215-224$.

Pfeffer, R.A. \& G.W. Tribbles. 1985. Hurricane effects on an aquarium fish fishery in the Hawaiian islands. Proc. $5^{\text {th }}$ Int. Coral Reef Symp., Thaiti 3: 331-336.

Ramírez-Granados, R. 1952. Estudio ecológico preliminar de las lagunas costeras cercanas a Acapulco, Gro. Rev. Soc. Mex. Hist. Nat. 13: 199-218.

Robins, C.R. 1957. Effects of storms on the shallow-water fish fauna of southern Florida with new records of fishes from Florida. Bull. Mar. Sci. Gulf Caribb. 7: 266-275.

Rocha-Ramírez, A., R. Román-Contreras \& S. CházaroOlvera. 2002. Population structure and fecundity of Anopsilana oaxaca Carvacho \& Haasmann, 1984 (Isopoda, Cirolanidae) of the Pacific coast of Mexico. Crustaceana 75: 97-109.

Román-Contreras, R. 1976. Contribución al conocimiento de la biología del "langostino" Macrobrachium tenellum (Smith, 1871) en algunas lagunas costeras de Guerrero, México. Tesis de licenciatura, UNAM, México D.F., México.

Román-Contreras, R. 1991. Ecología de Macrobrachium tenellum (Smith) (Decapoda, Palaemonidae) en la Laguna Coyuca, Guerrero, Pacífico de México. An. Inst. Cienc. Mar Limnol., UNAM 18: 109-121.

Román-Contreras, R. 1993a. Probopyrus pacificensis, a new parasite species (Isopoda: Bopyridae) of Macrobrachium tenellum (Smith, 1871) (Decapoda: Palaemonidae) of the Pacific Coast of Mexico. Proc. Biol. Soc. Wash. 106: 689-697.
Román-Contreras, R. 1993b. Aspectos biológicos de Probopyrus pacificensis Román-Contreras, 1993, parásito del langostino Macrobrachium tenellum (Smith, 1871) en la laguna Coyuca, Guerrero, México. Tesis de doctorado, UNAM, México D.F., México.

Román-Contreras, R. 2004. The genus Probopyrus Giard and Bonnier, 1888 (Crustacea: Isopoda: Bopyridae) in the eastern Pacific with seven new records for Mexico, p. 153-168. In M.E. Hendrickx (ed.). Contribuciones al Estudio de los Crustáceos del Pacífico Este 3. Instituto de Ciencias del Mar y Limnología, UNAM, México D.F., México.

Stoddart, D.R. 1962. Catastrophic storm effects on the British Honduras reefs and cays. Nature 196: 512-515.

Stuardo, J. \& A. Martínez. 1975. Resultados generales de una prospección de los recursos biológicos y pesqueros del sistema lagunar costero de Guerrero, México. Acta Politéc. Mex. 17: 99-115.

Stuardo, J. \& M. Villarroel. 1976. Aspectos ecológicos y distribución de los moluscos en las lagunas costeras de Guerrero, México. An. Centro Cienc. Mar y Limnol., UNAM 3: 65-92.

Vermeer, D.E. 1963. Effects of the hurricane Hattie, 1961, on the cays of British Honduras. Zeit. Geomorphol. 7: $332-354$.

Williams, A.H. 1984. The effects of hurricane Allen on back reef population of Discovery Bay, Jamaica. J. Exp. Mar. Biol. Ecol. 75: 233-243.

Yáñez-Arancibia, A. 1976. Observaciones sobre Mugil curema Valenciennes en áreas naturales de crianza, México. Alimentación, crecimiento, madurez y relaciones ecológicas. An. Centro Cienc. Mar y Limnol., UNAM 3: 94-132.

Yáñez-Arancibia, A. 1978a. Patrones ecológicos y variación cíclica de al estructura trófica de las comunidades nectónicas en lagunas costeras del Pacífico de México. Rev. Biol. Trop. 26: 191-218.

Yáñez-Arancibia, A. 1978b. Taxonomía, ecología y estructura de las comunidades de peces en lagunas costeras con bocas efímeras del Pacífico de México. Centro Cienc. Mar Limnol., UNAM Publ. Esp. 2: 1-306.

Yoshioka, P.M. \& B.B. Yoshioka. 1987. Variable effects of hurricane David on the shallow water gorgonian of Puerto Rico. Bull. Mar. Sci. 40: 132-144. 